https://doi.org/10.22319/rmcp.v10i4.4959

Artículo

\title{
Endoparásitos de Odocoileus virginianus y Mazama temama bajo cautiverio en Veracruz, México
}

Cristina Salmorán-Gómez ${ }^{\text {a }}$

Ricardo Serna-Lagunes ${ }^{a *}$

Norma Mora Collado ${ }^{a}$

Dora Romero-Salas ${ }^{b}$

Dulce María Ávila-Nájera ${ }^{c}$

Pedro Zetina-Córdoba ${ }^{c}$

${ }^{a}$ Universidad Veracruzana, Facultad de Ciencias Biológicas y Agropecuarias, región Orizaba-Córdoba, Unidad de Manejo y Conservación de Recursos Genéticos. Josefa Ortiz de Domínguez S/N, Col. Centro, Peñuela. 94945 Amatlán de los Reyes, Veracruz, México.

${ }^{\mathrm{b}}$ Universidad Veracruzana, Facultad de Medicina Veterinaria y Zootecnia, Veracruz, México.

c Universidad Politécnica de Huatusco. Unidad Académica de Biotecnología y Agroindustrial. Huatusco, Veracruz, México.

* Autor de correspondencia: rserna@uv.mx

\section{Resumen:}

El estudio de la parasitología de animales silvestres de importancia económica en cautiverio es relevante, ya que la parasitosis se traduce en pérdidas por disminución de productividad, aumento de gastos veterinarios, infecciones secundarias y pérdida de ejemplares. El objetivo de este trabajo fue determinar la prevalencia y abundancia de la diversidad de endoparásitos presentes en Odocoileus virginianus y Mazama temama en cautiverio. Durante la temporada de lluvias y secas, se recolectaron 60 muestras fecales de seis ejemplares de $O$. virginianus y cuatro de $M$. temama de diferente edad y sexo; se usó la técnica de flotación con solución saturada de azúcar, para recolectar e identificar 
los parásitos mediante comparación anatómica. Se identificaron siete géneros de parásitos: Ascaris sp., Eimeria sp., Estrongilido sp., Strongyloides sp., Parascaris sp., Paragonimus sp., y Taenia sp., siendo los dos primeros con mayor abundancia en ambos cérvidos. La prueba de $X^{2}$ determinó que la presencia de endoparásitos está asociada al sexo y a la especie de venado: machos y hembras de cada especie de venado, presentaron distinta prevalencia parasitaria. Parascaris sp. y Paragonimus sp., fueron parásitos que se encontraron solamente en $O$. virginianus. No se encontraron diferencias significativas $(P>0.05)$ entre la abundancia de parásitos entre las épocas de lluvias y secas; el género Ascaris fue significativamente más abundante con respecto a otros parásitos $(P<0.05)$. Esta información sirve para el control y prevención de parásitos de ungulados en cautiverio de importancia económica y para su conservación.

Palabras clave: Ascaris sp., Cérvidos, Parasitosis, Técnica de flotación, Zoonosis.

Recibido: 28/06/2018

Aceptado: 05/10/2018

\section{Introducción}

Desde hace un siglo, se ha estudiado la ecología de enfermedades y parasitosis en fauna silvestre, con especial énfasis en especies cinegéticas y de consumo ${ }^{(1,2)}$. Este tema en las últimas décadas se ha convertido en un campo de estudio desde un enfoque zoosanitario, ya que pueden desarrollarse enfermedades zoonóticas que afectan a especies domésticas y al ser humano, provocando la muerte de poblaciones silvestres y en cautiverio ${ }^{(3,4,5,6)}$.

El venado cola blanca (Odocoileus virginianus), es una especie de alta demanda para diversos usos y fines cinegéticos ${ }^{(7,8)}$. En esta especie, se ha descrito la diversidad de parásitos que causan enfermedades infecciosas ${ }^{(5)}$, lo que afecta su comportamiento, reproducción e inclusive la morbilidad y mortalidad ${ }^{(2,9,10)}$. Por su parte, en el venado temazate (Mazama temama), especie emblemática de los ecosistemas tropicales, son escasos los estudios sobre su parasitosis ${ }^{(1)}$ y mínimo conocimiento de las condiciones que deben mantenerse en cautiverio, que permitan el mantenimiento de sus poblaciones.

De manera general, los parásitos que más abundan en animales domésticos son protozoarios, helmintos, artrópodos y pentastómidos ${ }^{(12)}$. En el caso particular de los cérvidos, las enfermedades más comunes son causadas por virus, bacterias, cuadros infecciosos y parasitosis ${ }^{(2)}$. Dentro de las patologías más importantes que afectan la salud de los venados, figura la parasitosis gastrointestinal, la cual es provocada principalmente por helmintos y protozoarios ${ }^{(13)}$. El clima ${ }^{(14)}$, presencia o ausencia de hospederos intermediarios, composición del suelo, tipo de vegetación y calidad de agua, son factores 
que principalmente influyen en la prevalencia de parásitos ${ }^{(12)}$. Estudios realizados en poblaciones silvestres de $O$. virginianus muestran que la mortalidad de venados causada por parásitos gastrointestinales es alrededor del $2.7 \%{ }^{(15)}$.

En México, las Unidades de Manejo para la Conservación de la Vida Silvestre (UMA) son estrategias que se han implementado para el manejo con fines de conservación y aprovechamiento de la fauna silvestre; en su modalidad de manejo intensivo, animales mal manejados por la aplicación de protocolos zoosanitarios inadecuados, se traduce en pérdidas por disminución de su reproducción y productividad ${ }^{(16)}$, mayor incidencia de infecciones secundarias, ya que aumentan las lesiones en el tracto digestivo, reacciones anafilácticas, anemia, hasta llegar a la muerte del ejemplar; así mismo, incrementan la posibilidad de convertirse en enfermedades zoonóticas y se potencializa el riesgo de contagio entre la fauna silvestre y animales de producción pecuaria ${ }^{(3,17)}$, si no se aplican adecuadas medidas de prevención y mitigación ${ }^{(18)}$. Al respecto, los programas de manejo de cérvidos deben considerar la prevención y control de las enfermedades infecciosas y parasitarias más comunes, para garantizar la viabilidad poblacional y el éxito en los sistemas de producción ${ }^{(2)}$.

Dentro de las UMA, los estudios y la información generada de la parasitosis de fauna silvestre en cautiverio es precaria ${ }^{(15,19,20)}$ y los planes de manejo para la producción exitosa de cérvidos en UMA in situ y ex situ distan de su cometido ${ }^{(21)}$. En este sentido, es necesario generar conocimiento de los parásitos que afectan la salud de las poblaciones de cérvidos ex situ. Por lo anterior, el objetivo de la presente investigación fue conocer la prevalencia, diversidad de endoparásitos y la abundancia parasitaria que presenta una población de $O$. virginianus y $M$. temama en cautiverio.

\section{Material y métodos}

\section{Área de estudio.}

El presente trabajo se realizó en la UMA "El Pochote” en modalidad intensiva, registrada ante la Secretaría de Medio Ambiente y Recursos Naturales (SEMARNAT) con clave: UMA-IN-CR-0122-VER/og. Se ubica en el municipio de Ixtaczoquitlán, Veracruz, México, entre las coordenadas extremas $18^{\circ} 52^{\prime} 13.70^{\prime \prime} \mathrm{N}$ y $97^{\circ} 02^{\prime} 59.97^{\prime \prime} \mathrm{O}$, a 1,137 msnm. El objetivo principal de esta UMA es la conservación y reproducción de O. virginianus y M. temama.

La región presenta un clima semi-cálido húmedo (Cwa) con abundantes lluvias en verano, temperatura anual que oscila entre $\operatorname{los} 18$ a $24{ }^{\circ} \mathrm{C}$ y con precipitación pluvial media anual de 1,900 a 2,600 mm. La selva mediana subperennifolia y el acahual, son los principales tipos de vegetación alrededor de la UMA El Pochote ${ }^{(22)}$. 


\section{Ejemplares de cérvidos en estudio}

Se trabajó con seis individuos de $O$. virginianus: tres hembras (2, 3 y 5 años de edad) y tres machos (3, 4 y 9 años) y con cuatro ejemplares de $M$. temama: dos hembras (2 y 4 años) y dos machos (ambos de 3 años), todos aparentemente sanos y con buena conformación corporal. Un año antes de iniciar el muestreo, los venados se trataron con Hemoplex ${ }^{\circledR}$, un tónico general a una dosis de $2 \mathrm{ml}$ por cada $10 \mathrm{~kg}$ de peso; además se suministró Catosal ${ }^{\circledR}$ vía muscular como estimulante metabólico. Los ejemplares de ambas especies de venados, se mantuvieron en diferentes encierros con un tamaño de 30 $\mathrm{m}$ de longitud por $12.5 \mathrm{~m}$ de ancho, con malla venadera como delimitación y con una separación entre encierros de $50 \mathrm{~m}$; los corrales presentan dos bebederos, techumbre para cubrirlos de la intemperie, se colocó una malla con $80 \%$ de sombra a la altura de la visión de los venados (de 60 a $1.2 \mathrm{~cm}$ ) para evitar el contacto visual con otros venados; diariamente, se realizó la limpieza de heces. Los animales se alimentaron a las $0800 \mathrm{~h}$ con una ración diaria de alfalfa (20\% $2 \mathrm{~kg}$ por animal) y alimento balanceado para ovino (80\% $4 \mathrm{~kg}$ por animal) que contenía proteína cruda (34 \%), grasa (2\%), fibra cruda (5\%), cenizas (17\%) y humedad (13\%); se les ofreció agua ad libitum.

\section{Muestreo de heces}

La colecta de los grupos fecales se realizó en dos épocas: lluvias (septiembre-noviembre) y secas (marzo-mayo), dado que hay variación en la incidencia y abundancia de parásitos entre épocas ${ }^{(23)}$. En cada periodo de muestreo, mensualmente se obtuvieron las muestras fecales de todos los ejemplares, aprovechando la primera defecación espontánea de los venados, la cual se presentó entre las 0600 y 0900 h. Con guantes de látex, se tomó la porción de la excreta que no se encontraba en contacto directo con el suelo; las muestras se depositaron en bolsas plásticas con cierre hermético con su respectiva ficha de colecta, se mantuvieron en una hielera térmica a $4{ }^{\circ} \mathrm{C}$. Las muestras se transportaron al laboratorio de Microscopía Óptica de la Facultad de Ciencias Biológicas y Agropecuarias, región Orizaba-Córdoba, Universidad Veracruzana, para su posterior análisis. Al final del muestreo, se recolectaron 60 muestras de heces de ambas especies de venados, 30 muestras fueron recolectadas en el periodo de lluvias y 30 muestras en secas durante 2016 y 2017, respectivamente. 


\section{Análisis de muestras fecales}

Para el análisis parasitoscópico, se utilizó la técnica de flotación en solución saturada de azúcar, la cual consiste en la separación de partículas de mayor y menor densidad. De la solución, se colectaron huevos ligeros e individuos completos de parásitos que se fijaron en portaobjetos, para su posterior identificación morfológica ${ }^{(24)}$.

\section{Identificación morfológica y abundancia de endoparásitos}

Los parásitos encontrados se identificaron mediante comparación de sus características anatómicas con los reportados en los Catálogos Parasitológicos ${ }^{(25)}$, libros de parasitología y de enfermedades parasitarias de animales domésticos ${ }^{(12)}$. Se identificó el género del taxón de los ooquistes con base en el número de esporozoítos que presentaron ${ }^{(26)}$. La abundancia se consideró como el número de endoparásitos presentes por cada cérvido hospedero, ya que es una medida indirecta de la prevalencia ${ }^{(20,27)}$.

\section{Análisis estadístico de datos}

Para este trabajo, las especies de venados ( $O$. virginianus y $M$. temama), el sexo (machos y hembras) y la temporada (secas y lluvias) fungieron como las fuentes de variación, y como variable respuesta fue la abundancia de parásitos de cada cérvido hospedero, ya que ésta se considera como un indicador de infección por parásitos nematodos ${ }^{(27)}$. Se calculó la prevalencia (\%) por sexo, por especie de cérvido y se obtuvieron los estadísticos descriptivos de la abundancia por cada fuente de variación. Se aplicó una prueba de Kruskall-Wallis junto con la prueba de comparación de medias LSD (Least significant difference $)$ de Fisher $(\alpha=0.05)$ con el objetivo de determinar qué especie de parásito fue más abundante en determinada especie de venado, sexo y temporada (lluvias o secas). Adicionalmente, se analizó la asociación de la abundancia de parásitos con respecto a la especie de cérvido y el sexo del animal, con una prueba de Ji cuadrada. 


\section{Resultados}

Se identificaron siete géneros de endoparásitos: Ascaris sp., Eimeria sp., Estrongilido sp., Paragonimus sp., Parascaris sp., Strongyloides sp. y Taenia sp., con diferentes abundancias (Figura 1), que variaron en la época de lluvias y secas y en la especie de cérvido estudiado (Cuadro 1). De los 10 ejemplares bajo estudio, el 100\% presentaron endoparásitos, siendo Parascaris sp., Paragonimus sp., y Taenia sp., los parásitos gastrointestinales prevalentes en $O$. virginianus y ausentes en $M$. temama.

Figura 1: Abundancia de endoparásitos en O. virginianus y M. temama en la UMA El Pochote. Letras diferentes en las barras representan diferencias significativas

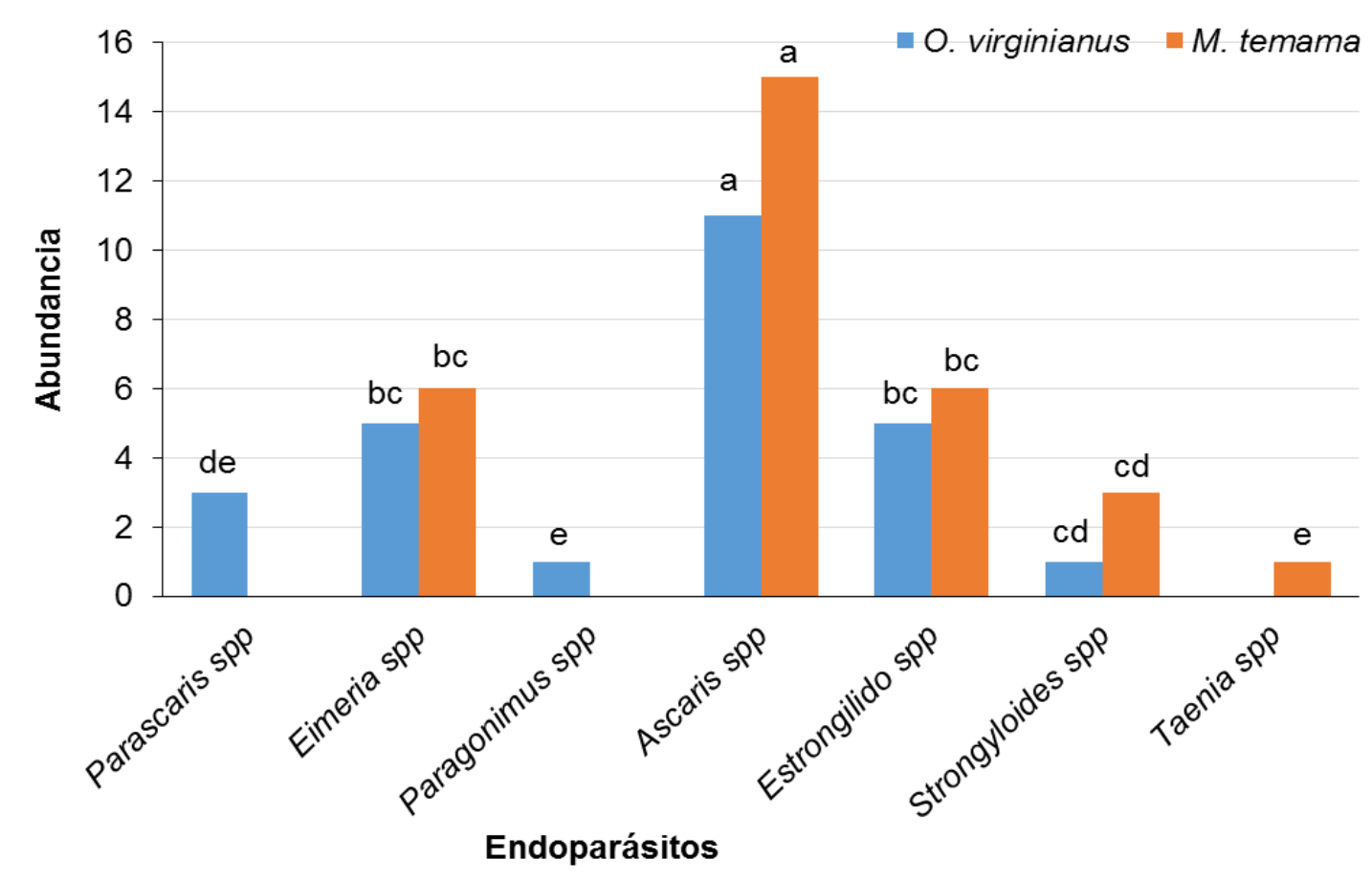


Cuadro 1: Géneros identificados y abundancia promedio de endoparásitos en O. virginianus y M. temama durante la época de lluvias y de secas en la UMA El Pochote, Veracruz, México

\begin{tabular}{|c|c|c|c|c|}
\hline Época & Especie & Parásito & Abundancia promedio & DE \\
\hline \multirow{9}{*}{$\frac{\stackrel{\Xi}{\Xi}}{\Xi}$} & \multirow{5}{*}{ 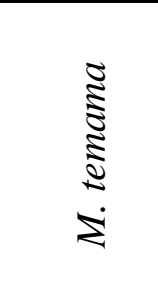 } & Ascaris sp. & 2.00 & 1.4 \\
\hline & & Eimeria sp. & 1.67 & 1.7 \\
\hline & & Estrongilido sp. & 1.67 & 1.2 \\
\hline & & Strongyloides sp. & 0.67 & 1.1 \\
\hline & & Taenia sp. & 0.33 & 0.8 \\
\hline & \multirow{4}{*}{ 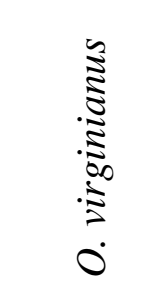 } & Ascaris sp. & 2.33 & 2.0 \\
\hline & & Eimeria sp. & 1.67 & 1.2 \\
\hline & & Paragonimus sp. & 0.33 & 0.8 \\
\hline & & Parascaris $s p$. & 0.33 & 0.8 \\
\hline \multirow{8}{*}{$\begin{array}{l}\tilde{J} \\
\tilde{J} \\
\tilde{E}\end{array}$} & \multirow{3}{*}{ 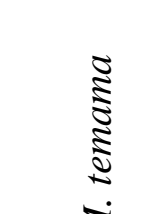 } & Ascaris sp. & 3.00 & 0.0 \\
\hline & & Eimeria sp. & 0.33 & 0.8 \\
\hline & & Estrongilido sp. & 0.33 & 0.8 \\
\hline & $\dot{\Sigma}$ & Strongyloides sp. & 0.33 & 0.8 \\
\hline & \multirow{4}{*}{ 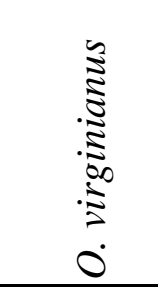 } & Ascaris sp. & 1.33 & 1.1 \\
\hline & & Estrongilido sp. & 1.67 & 1.7 \\
\hline & & Parascaris sp. & 0.67 & 0.8 \\
\hline & & Strongyloides sp. & 0.33 & 0.8 \\
\hline
\end{tabular}

$\mathrm{DE}=$ desviación estándar.

La época y la especie de cérvido no tuvo un efecto significativo $(P>0.05)$ con respecto a la abundancia de los parásitos (Cuadro 2), pero se detectaron diferencias significativas en la abundancia de los géneros de endoparásitos identificados $(P<0.05)$, siendo Ascaris sp., la especie más abundante (Cuadro 3). 
Cuadro 2: Efecto de la época del año (secas vs lluvias), la especie de cérvido hospedero (O. virginianus y M. temama) y el género de endoparásitos

\begin{tabular}{lccccc}
\hline & SC & GL & CM & F & P-valor \\
\hline Modelo & 13.06 & 8 & 1.63 & 2.98 & 0.007 \\
Época & 0.14 & 1 & 0.14 & 25 & 0.619 \\
Especie de cérvido & 1.07 & 1 & 1.07 & 1.95 & 0.167 \\
Parásito & 11.86 & 6 & 1.98 & 3.61 & $0.004^{*}$ \\
Error & 31.2 & 57 & 0.55 & & \\
Total & 44.26 & 65 & & & \\
\hline
\end{tabular}

$\mathrm{SC}=$ suma de cuadrados; GL grados de libertad; $\mathrm{CM}=$ cuadrado medio; $\mathrm{F}=$ valor de tablas ; $P$-valor $=$ valor de significancia

Cuadro 3: Abundancia promedio comparada por género de endoparásito en las dos especies de venado

\begin{tabular}{lcccc}
\hline Endoparásito & N & Promedio & EE & Diferencias \\
\hline Taenia sp. & 6 & 0.17 & 0.57 & $\mathrm{a}$ \\
Paragonimus sp. & 6 & 0.17 & 0.57 & $\mathrm{a}$ \\
Strongyloides $\mathrm{sp}$. & 12 & 0.33 & 0.4 & $\mathrm{a}$ \\
Parascaris sp. & 6 & 0.5 & 0.57 & $\mathrm{a}$ \\
Estrongilido sp. & 12 & 0.92 & 0.4 & $\mathrm{a}$ \\
Eimeria sp. & 12 & 0.92 & 0.4 & $\mathrm{a}$ \\
Ascaris sp. & 12 & 2.17 & 0.4 & $\mathrm{~b}^{*}$ \\
\hline
\end{tabular}

$\mathrm{N}=$ tamaño muestral; $\mathrm{EE}=$ error estándar; *diferencias a un $\alpha=0.05$.

Eimeria sp fue el género de parásito cuya presencia estuvo asociada a machos de $M$. temama y a hembras de $O$. virginianus $\left(X^{2}=8.57\right.$, g.1. $\left.1 ; P=0.0034\right)$. Taenia sp., y Paragonumus sp., se presentaron en un macho de $M$. temama y en una hembra de $O$. virginianus, respectivamente $\left(X^{2}, P<0.05\right)$, mientras que Parascaris sp., Ascaris sp., Estrongilido sp., Strongyloides sp., no mostraron asociación $\left(X^{2}, P>0.05\right)$ con respecto al sexo o a la especie de cérvido.

\section{Discusión}

Se identificaron siete géneros de endopárasitos en $O$. virginianus y $M$. temama, por lo que este estudio representa el primer reporte de estos endoparásitos en UMAs in situ o ex situ que se encuentran localizadas en el territorio veracruzano. En ambas temporadas y en los dos cérvidos estudiados, se registraron los géneros Ascaris sp., y Eimeria sp., mientras que Taenia sp., solamente se registró para la época de lluvias $(P>0.05)$ en M. temama. 
En diversos trabajos realizados, con un número variable de entre 20 y 200 muestras de excretas analizadas en diferentes épocas del año (secas, transición y lluvias), se describieron ocho géneros de parásitos; de ellos, los que destacan en común con nuestro estudio y los que provocan una mayor abundancia parasitaria fueron Eimeria sp., y Strongyloides $\mathrm{sp}^{(20,27,28)}$. En un estudio donde se recolectaron aproximadamente 1,000 muestras de heces de $O$. virginianus de tres confinamientos en un periodo de un año ${ }^{(15)}$, identificaron siete géneros de endoparásitos, de los cuales Eimeria y Strongyloides coinciden con lo reportado en los trabajos previamente citados y con el presente estudio.

En los ciervos de la UMA bajo estudio, Ascaris sp., fue el género con prevalencia en ambos cérvidos hospederos, de acuerdo a los análisis estadísticos existen diferencias significativas de su abundancia con respecto a los otros géneros de parásitos identificados. Un resultado similar se ha reportado con los parásitos de la Familia Ascarididae, que usualmente se encuentran en el intestino de peces, anfibios, reptiles, aves y mamíferos, pero en las especies que más daño causan son en especies de producción pecuaria, parasitando cerdos, equinos, ganado vacuno, aves de corral, perros, gatos y mamíferos silvestres como zorros ${ }^{(27)}$. Por otro lado, en primates: Alouatta fusca y A. seniculus se ha reportado la presencia de huevos de nematodos de Ascaris sp., adjudicado a su cuidador como la probable fuente de contaminación antropozoonótica ${ }^{(29)}$. En el caso de las especies del género Eimeria sp., que parasita principalmente a mamíferos, son comúnmente parásitos del canal digestivo del hospedero y se arraigan en las células epiteliales las cuales destruyen, causando la enfermedad conocida como coccidiosis ${ }^{(25)}$.

Los ungulados mantenidos en cautiverio presentan un mayor número de endoparásitos comparado con los ungulados de vida libre ${ }^{(13)}$; lo que puede deberse a que los animales mantenidos en cautiverio generalmente consumen alimentos brindados por el ser humano sin implementar cuidados sanitarios, en los encierros puede haber mayor humedad, formación de charcas, fuga de agua de bebederos, etc., incrementando las fuentes de riesgo de transmisión parasitaria ${ }^{(30)}$. Aunado a esto, el estrés del cautiverio puede disminuir la capacidad inmunológica y propiciar el surgimiento de la parasitosis y una mayor diversidad y abundancia parasitaria ${ }^{(31)}$.

Cuando se compararon los tipos de parásitos y su abundancia entre épocas del año encontrados en este trabajo con lo reportado en otros estudios, estos tampoco detectaron diferencias significativas entre los meses de muestreo ${ }^{(20,28)}$. Estos resultados se atribuyen a que los cambios ambientales en las dos temporadas, condicionan el ambiente favorable para la transmisión parasitaria; en caso de necesitar desarrollo fuera del hospedero, debe existir la presencia de hospederos intermediarios, favoreciendo o impidiendo el desarrollo parasitario en los cérvidos ${ }^{(32)}$.

Los animales silvestres son hospederos de diferentes parásitos sin que estos causen síntomas de enfermedad ${ }^{(33)}$, ya que puede mantenerse un equilibrio. Al respecto, algunos autores $^{(34)}$, mencionan que existen factores que debilitan el sistema inmune de los hospederos, tales como la edad, desnutrición y estrés, entre otras causas que aumentan el riesgo para que el organismo se sobre-parasite. Estudios demuestran que el contagio de 
endoparásitos entre especies silvestres y domésticos puede ser peligroso ${ }^{(35)}$. La abundancia y riqueza de parásitos puede variar entre especies de cérvidos ${ }^{(36)}$, lo cual está relacionado al hábitat, otras especies con las que coexiste, tamaño y características de los encierro y densidad poblacional ${ }^{(37)}$; futuros estudios deben considerar las características del encierro de las UMA, para detectar los factores de riesgo asociados a la parasitosis.

La aparición y dinámica de parásitos a lo largo del año, puede estar influida por el sexo del huésped. La prevalencia de parásitos con respecto al sexo de los animales está ligada a características del individuo como la edad y la condición corporal ${ }^{(38)}$. En Alces alces ${ }^{(39)}$ y en $O$. virginianus $^{(40)}$ se ha reportado que ejemplares adultos tienen una mayor carga parasitaria que los sub-adultos, en la época de celo y sin celo, respectivamente. En este estudio, Eimeria sp., y Paragonumus sp., fueron prevalentes en hembras de O. virginianus, lo que coincidió con la temporada de apareamientos, contagiadas posiblemente por los sementales. El macho de M. temama que estuvo parasitado por Taenia sp., su contagio posiblemente ocurrió por alta humedad que prevalece en su encierro, por las hojas de los árboles que caen al corral o por los brotes de hierba que este ejemplar ingiere. Sin embargo, se requiere seguir estudiando la parasitología de M. temama para fortalecer los programas de manejo en cautiverio, y contribuir a su conservación, como en los casos exitosos de otros ungulados (p. ej. Gazella gazella) ${ }^{(41)}$.

\section{Conclusiones e implicaciones}

Este estudio proporciona datos básicos sobre la prevalencia y diversidad de parásitos gastrointestinales en $O$. virginianus y $M$. temama bajo cautiverio. Se registraron siete géneros de parásitos en ambas especies de cérvidos, ambas especies de venados podrían ser tratadas con desparasitantes específicos que eviten una carga parasitaria excesiva, que cause la morbilidad o mortalidad de los hospederos. Por los resultados obtenidos, la época de secas es la mejor estación para aplicar un tratamiento antiparasitario. Ascaris sp., fue abundante en ambos cérvidos, seguida de Estrongilido sp., y Eimeria sp. Estos hallazgos resaltan la importancia de identificar los factores de riesgo de parasitosis en la fauna silvestre en cautiverio, para optimizar las estrategias de prevención y mitigación. Este estudio tiene implicaciones en la conservación y manejo de $O$. virginianus y $M$. temama bajo cautiverio, así como la prevención de enfermedades zoonóticas que puedan afectar las poblaciones tanto de animales silvestres como de especies domésticas y a su vez tenga implicaciones económicas para los productores. 


\section{Agradecimientos}

A Arantxa Penagos de la Llave, por la ayuda técnica recibida y por sus facilidades otorgadas en la UMA El Pochote. A Carlos Manuel Galán Páez por su apoyo en el proceso de laboratorio. A la Unidad de Manejo y Conservación de Recursos Genéticos de la Facultad de Ciencias Biológicas y Agropecuarias, por las facilidades bioinformáticas proporcionadas.

\section{Literatura citada:}

1. Wells K, Gibson DI, Clark NJ, Ribas A, Morand S, McCallum HI. Global spread of helminth parasites at the human-domestic animal-wildlife interface. Glob Chang Biol 2018;24(7):3254-3265.

2. Campbell T, VerCauteren KC. Diseases and parasites of White-Tailed Deer. In: Hewitt DG editor. Biology and management of White-Tailed Deer. USDA National Wildlife Research Center-Staff Publications. 2011:219-249.

3. Wobeser GA. Investigation and management of disease in wild animals. New York, USA: Plenum Press; 1994.

4. Schultz SR, Barry RX, Johnson MK, Miller JE, Forbes DA. Effects of feed plots on fecal egg counts of white-tailed deer. Small Rumin Res 1994;13(1):93-97.

5. Medina-Vogel G. Ecología de enfermedades infecciosas emergentes y conservación de especies silvestres. Arch Med Vet 2010;42(1):11-24.

6. Gallina S, Escobedo-Morales LA. Análisis sobre las Unidades de Manejo (UMAs) de ciervo rojo (Cervus elaphus Linnaeus, 1758) y wapiti (Cervus canadensis Erxleben, 1777) en México: problemática para la conservación de los ungulados nativos. Trop Conserv Sci 2009;2(2):251-265.

7. Davidson WR, McGhee MB, Nettles VF, Chappell LC. Haemonchosis in whitetailed deer in the southeastern United States. J Wild Dis 1980;16(4):499-508.

8. Mandujano S, Pérez TD, Escobedo LA, Yañez C, González A, Pérez LA, Ramos MI. Venados: animales de los dioses. Veracruz, México: Secretaria de Educación de Veracruz; 2010.

9. Conti JA, Howerth EW. Osteragiosis in a white-tailed deer due to Ostertagia. J Wild Dis 1987;23(1):159-162.

10. Suzán G, Galindo F, Ceballos G. La importancia del estudio de enfermedades en la conservación de fauna silvestre. Vet México 2000;31(3):223-230. 
11. Mandujano S. Análisis bibliográfico de los estudios de venados en México. Acta Zool Mex 2004;20(1):211-251.

12. Quiroz RH. Parasitología y enfermedades parasitarias de animales domésticos. México: Ed. Limusa; 2012.

13. Mukul-Yerves JM, Zapata-Escobedo MDR, Montes-Pérez RC, Rodríguez-Vivas RI, Torres-Acosta JF. Parásitos gastrointestinales y ectoparásitos de ungulados silvestres en condiciones de vida libre y cautiverio en el trópico mexicano. Rev Mex Cienc Pecu 2014;5(4):459-469.

14. Patz JA, Graczyk TK, Geller N, Vittor AY. Effects of environmental change on emerging parasitic diseases. Int J Parasitol Parasites Wildl 2000;30(12-13):13951405.

15. Montes PRC, Rodríguez VRI, Torres AFJ, Ek PLG. Seguimiento anual de la parasitosis gastrointestinal de venados cola blanca, O. virginianus (Artiodactyla: Cervidae) en cautiverio en Yucatán, México. Rev Biol Trop 1998;46(39):821-827.

16. García RC. Parasitosis del Ciervo Ibérico Español. Madrid, España: Ministerio de Agricultura, Pesca y Alimentación. 2006.

17. Reissig EC, Massone AR, Iovanitti B, Gimeno, EJ, Uzal FA. A survey of parasite lesions in wild red deer (Cervus elaphus) from Argentina. J Wild Dis [in press] 2018; doi.org/10.7589/2017-06-136.

18. Hernández JA, Arroyo FL, Banilla R, Sánchez-Andrade R, Arias MS. Control biológico de parásitos en la ganadería, hongos del duelo. Zoocienc 2015;2(1):2-11.

19. Aguilar R. Gusanos parásitos de fauna silvestre. Algunas formas de estudio. Ciênc Cult 2008;15:55-61

20. Valdés VV, Saldaña A, Pineda VJ, Camacho JA, Charpentier CV, Cruz TA. Prevalencia de parásitos gastrointestinales en Odocoileus virginianus y Tayassu tajacu en cautiverio de la República de Panamá. Acta Zool Mex 2010;26(2):477480.

21. Gallina-Tessaro S, Hernández-Huerta A, Delfín-Alfonso CA, González-Gallina A. Unidades para la conservación, manejo y aprovechamiento sustentable de la vida silvestre en México (UMA). Retos para su correcto funcionamiento. Investigación Ambiental, Ciencia y Política Pública 2009;1(2):143-152.

22. Cházaro-Basáñez MJ. Exploraciones botánicas en Veracruz y estados circunvecinos. I. Pisos altitudinales de vegetación en el centro de Veracruz y zonas limítrofes con Puebla. Cienc Hombre 1992;10:67-115.

23. Quiroz RH, Figueroa J, Ibarra F, López M. Epidemiología de enfermedades parasitarias en animales domésticos. México: Facultad de Medicina Veterinaria y 
Zootecnia, Departamento de Parasitología, Universidad Nacional Autónoma de México. 2011.

24. Liébano HE, Flores CJ. Identificación de larvas infectantes de nematodos gastroentéricos en bovinos y ovinos de México. En: Centro Nacional de Investigaciones Disciplinarias en Parasitología Veterinaria Editor. Diagnóstico de las nematodosis gastrointestinal de los rumiantes en México: México; 1999:23-40.

25. Cordero M, Rojo F, Martínez A, Sánchez C, Hernández S, Navarrete J, Carvalho M. Parasitología Veterinaria. España: Mc Graw-Hill Interamericana; 1999.

26. Rodríguez-Vivas RI, Cob-Galera LA. Técnicas diagnósticas en parasitología veterinaria. Mérida, México: Universidad Autónoma de Yucatán. Mérida, México; 2005.

27. Mukul-Yerves JM, Pereida-Hoíl AJ, Rodríguez-Vivas RI, Montes-Pérez RC. Parasitosis gastrointestinal en venados cola blanca (Odocoileus virginianus yucatanensis) y temazate (Mazama temama) en condiciones de cautiverio en Yucatán, México. Bioagrociencias 2004;7(1): 33-37.

28. Barranco SG. Frecuencia de parásitos gastrointestinales en heces de venado cola blanca (Odocoileus virginianus) pertenecientes a unidades de manejo para la conservación de la vida silvestre (UMA) del estado de Morelos [tesis Licenciatura]. Toluca, México: Universidad Autónoma del Estado de México; 2016.

29. Beltrán-Saavedra L, Martín P, Gonzáles JL. Estudio coproparasitológico de mamíferos silvestres en cautiverio con destino a relocación en Santa Cruz, Bolivia. Vet Zootec 2008;3(1):51-60.

30. Konjević D, Janicki Z, Calmels P, Jan DS, Marinculić A, Šimunović M, Poljak Z. Evaluation of factors affecting the efficacy of treatment against Fascioloides magna in wild red deer population. Vet Ital 2018;54(1):33-39.

31. Martínez FA, Binda JL, Maza Y. Determinación de platelmintos por coprología en carnívoros silvestres. En: Universidad Nacional del Nordeste Editor. Comunicaciones científicas y tecnológicas. Argentina. 2005.

32. Xiao-Long H, Gang L, Yu-Ting W, Yi-Hua W, Tian-Xiang Z, Shuang Y, De-Fu H, Shu-Qiang L. Regional and seasonal effects on the gastrointestinal parasitism of captive forest musk deer. Acta Trop 2018;177:1-8.

33. Romero S, Ferguson B, Guiris D, González D, López S, Paredes A, Weber M. Comparative parasitology of wild and domestic ungulates in The Selva Lancandona, Chiapas, México. Proc Helminthol Soc Wash 2008;75(1):115-126.

34. Cañizales I, Guerrero R. Parásitos y otras enfermedades transmisibles de la fauna cinegética en Venezuela. En: Academia de ciencias físicas, matemáticas y naturales: Simposio de investigación y manejo de fauna silvestre en Venezuela. 2010:97-108. 
35. Höglund J, Christensson D, Holmdahl J, Mörner T, Osterman E, Uhlhorn H. The first record of the nematode Ashworthius sidemi in Sweden. Proc $21^{\text {st }}$ Int Conf of WAAVP. Ghent, Belgium; 2007.

36. Kowal J, Nosal P, Bonczar Z, Wajdzik M. Parasites of captive fallow deer (Dama dama L.) from southern Poland with special emphasis on Ashworthius sidemi. Ann Parasitol 2012;58(1):23-26.

37. Davidson KR, Kutz JS, Madslien K, Hoberd E, Handeland K. Gastrointestinal parasites in an isolated Norwegian population of wild red deer (Cervus elaphus). Acta Vet Scand 2014;56(1):56-59.

38. Horcajada-Sánchez F, Navarro-Castilla Á, Boadella M, Barja I. Influence of livestock, habitat type, and density of roe deer (Capreolus capreolus) on parasitic larvae abundance and infection seroprevalence in wild populations of roe deer from central Iberian Peninsula. Mammal Res 2018;63(2):213-222.

39. Grandi G, Uhlhorn H, Ågren E, Mörner T, Righi F, Osterman-Lind E, Neimanis A. Gastrointestinal parasitic infections in dead or debilitated Moose (Alces alces) in Sweden. J Wildl Dis 2018;54(1):165-169.

40. Heine KB, DeVries PJ, Penz CM. Parasitism and grooming behavior of a natural white-tailed deer population in Alabama. Ethol Ecol Evol 2017;29(3):292-303.

41. Ortiz J, de Ybáñez MR, Garijo MM, Goyena M, Espeso G, Abaigar T, Cano M. Abomasal and small intestinal nematodes from captive gazelles in Spain. J Helminthol 2001;75(4):363-365. 